\title{
Glucose and Insulin Levels are Increased in Obese and Overweight Mothers’ Breast-Milk
}

\author{
Suman Ahuja ${ }^{1}$, Mallory Boylan ${ }^{2}$, Sybil L. Hart ${ }^{3}$, Carmen Román-Shriver ${ }^{4}$, Julian E. Spallholz ${ }^{2}$, \\ Barbara C. Pence ${ }^{5}$, Barbara G. Sawyer ${ }^{6}$
}

${ }^{1}$ Department of Nutrition, Hospitality, and Retailing Lubbock, Texas, USA; ${ }^{2}$ Associate Professor of Nutrition, Hospitality and Retailing Texas Tech University Lubbock, Texas, USA; ${ }^{3}$ Professor of Human Development and Family Studies, Texas Tech University Lubbock, Texas, USA; ${ }^{4}$ Associate Professor and Director, Nutrition and Dietetics University of Texas Health Sciences Center at San Antonio Laredo, Texas, USA; ${ }^{5}$ Professor and Associate Chair Department of Pathology Rm 1A-088 Texas Tech University Health Sciences Center 3601 4th Street MS 8115 Lubbock, Texas, USA; ${ }^{6}$ Professor of Laboratory Science and Primary Care Texas Tech University, Health Sciences Center Lubbock, Texas, USA.

Email: as2095@columbia.edu, mallory.boylan@ttu.edu,sybil.hart@ttu.edu,romanshrivec@uthscsa.edu, julian.spallholz@ttu.edu, barbara.pence@ttuhsc.edu, barbara.sawyer@ttuhsc.edu

Received March 9 ${ }^{\text {th }}, 2011$; Revised April 9 ${ }^{\text {th }}, 2011$; Accepted April 20 th $^{\text {th }} 2011$.

\begin{abstract}
Objective: To analyze the relationships between mothers' pre-pregnancy body mass index (BMI) and levels of glucose and insulin in breast-milk. Methods: Participants were 32 breast-feeding mothers who were assigned to either normal weight (Group 1: $n=21$, BMI between 18.5 and $24.9 \mathrm{~kg} / \mathrm{m}^{2}$ ) or overweight/obese (Group 2: $n=11, B M I \geq 25 \mathrm{~kg} / \mathrm{m}^{2}$ ) based on their pre-pregnancy BMI. Breast-milk samples were collected at six weeks postpartum. Glucose and insulin concentrations were measured in the breast-milk samples. To control for any extreme dietary influences on breast-milk glucose concentrations, mothers completed 24-hour food recalls. Results: Mean milk-glucose values of Groups 1 and 2 were $(32.6 \pm 21.8) \mathrm{mg} / \mathrm{dL}$ and $(51.9 \pm 20.5) \mathrm{mg} / \mathrm{dL}$ respectively. Milk-borne insulin values were $(4.5 \pm 7.6) \mu I U / \mathrm{mL}$ and $(30.1 \pm 56.3) \mu \mathrm{IU} / \mathrm{mL}$, respectively. Independent-samples $t$ tests found that glucose levels of Group 2 were greater than those of Group 1, $t(30)=2.43, p=0.02$; and milk-borne insulin was greater in Group 2 than in Group 1, $t(30)=2.075$, $p=0.05$. Mothers' pre-pregnancy BMI was found correlated with both breast-milk glucose $(r=0.483, p=0.005)$ and insulin ( $r=0.565, p=0.001)$. Conclusions: This study found that in comparison with normal weight mothers, overweight/ obese mothers have higher concentrations of glucose and insulin in their breast-milk. Further studies are needed to explain the underlying mechanisms, and possible consequences of infant exposure to higher levels of glucose and insulin in breast-milk.
\end{abstract}

Keywords: Breast-Milk, Glucose, Insulin, Obesity

\section{Background}

Breast-feeding is considered the ideal and preferred form of infant feeding. Among the numerous benefits of breastfeeding, studies find that breast-fed infants are leaner and better protected from obesity as they age [1,2]. Beginning in early childhood and progressing in later life, offspring of obese and diabetic mothers are at increased risk for developing overweight/obesity and impaired glucose tolerance (IGT) [3]. Greater weight gain among breast-fed infants of obese and diabetic mothers is poorly understood. In utero exposure to high concentrations of glucose and insulin from a diabetic mother can program adult insulin resistance, and increase the risk of obesity in later life. Some have speculated that even a slight increase in glucose levels in the breast-milk of a diabetic mother can have long-term repercussions, such as, increased weight gain and altered metabolism [3].

Elevated plasma glucose during pregnancy has been associated with increased fat mass in offspring, which according to Catalano et al. [4], poses risk of obesity in early childhood that may even continue through adulthood. A recent study reported that when present in milk of a diabetic lactating mother, increased concentrations of glucose and insulin may predispose her infant to develop obesity in adulthood [3]. The possible link between 
risk of infant obesity and exposure to a diabetic mother's breast-milk [3] calls for investigative attention with respect to characteristics of breast-milk that might play a role in the expression of this association. Thus, the present study focused on variations in levels of glucose and insulin in breast-milk. In particular, we explored whether glucose and insulin concentrations in the breast- milk of obese mothers are greater than those of normal weight mothers.

\section{Methods and Procedures}

\subsection{Participants and Population}

The Texas Tech University Health Science Center Institutional Review Board approved the study protocol, questionnaires, inclusion/exclusion criteria, and consent form. Pregnant women were recruited from a local university affiliated hospital while in their third trimester. Participant screening included collection of demographic data and patient medical history. During the initial screening and recruitment phase, 82 pregnant women responded. However, only 32 women met eligibility criteria, including no use of drugs, alcohol, and medications, and absence of chronic diseases such as type 1,2, and gestational diabetes, or any potential pregnancy related complications that may have interfered with the results of this study.

All 32 eligible participants had normal, full-term pregnancies and were breastfeeding exclusively when followed-up after delivery. All participants recruited for the study had normal blood glucose levels and absence of gestational diabetes. They also had normal glucose tolerance when tested during pregnancy. Subjects were assigned to one of two groups based on their pre-pregnancy BMI, which was obtained through patient medical records. Quetelet's body mass index (BMI) was calculated by dividing weight in kilograms by height squared in meters $\left(\mathrm{kg} / \mathrm{m}^{2}\right)$. Non-obese lactating mothers with a pre-pregnancy BMI between 18.5 to $24.9 \mathrm{~kg} / \mathrm{m}^{2}$ comprised Group $1(n=21)$. Group $2(n=11)$ was composed of both overweight and obese lactating mothers as defined by a pre-pregnancy BMI of $\geq 25.0 \mathrm{~kg} / \mathrm{m}^{2}$.

\subsection{Breast-Milk Sample Collection and Analyses of Milk Glucose and Insulin Concentrations}

At six weeks postpartum, milk samples were collected. To help minimize variation in glucose and insulin concentrations, and to control for biases across groups (with respect to time of day milk samples were collected, and meal consumption by the mother), all milk samples were collected in the morning between 9:00 a.m. and 11:00 a.m. Milk samples were collected immediately after the infant had terminated nursing on one breast on account of satiation. Contents of an entire breast were collected in a two ounce sterile tube attached to an electric breast pump (Medela, Inc., McHenery, IL). Milk was divided into aliquots and stored in polypropylene tubes and frozen at $-20^{\circ} \mathrm{C}$ until analysis.

\subsection{Dietary Assessment}

To control for possible confounding variables, specifically mothers' diet, on the amount of insulin and glucose in breast-milk, nutrient intakes for all subjects were assessed using a USDA approved 5-step automated multiple-pass 24-hours diet recall [5], and a food frequency questionnaire (FFQ) [6]. These were administered by a trained member of the research team who conducted dietary interviews prior to sample collection.

\subsubsection{5-Step Automated Multiple Pass 24-Hours Dietary-Recall Method}

This instrument was designed to address the problem of under-reporting food intake [7]. To overcome this type of instrument threat, the USDA improvised on the current 24-hour diet recall method and designed the 5-step automated multiple pass 24-hour dietary-recall method. This method was designed to aid in effective diet recall while reducing respondent burden [5,7]. Data obtained through the 5-step automated multiple pass 24-hours dietary-recall method were analyzed using a dietary analysis online tool from Nutrition Data (ND), Nutrition Facts and Calorie Counter [8]. Source of information provided on the Nutrition Data Website is generated from the USDA [8].

\subsubsection{Food Frequency Questionnaire (FFQ)}

Participating mothers also completed an interviewer-administered, modified version of the 72-item Johns Hopkins Weight Management Center FFQ [6]. This questionnaire obtains information on frequency of consuming meat and meat products, starches, fruits and vegetables, dairy products, combination foods, fats, oils, and sugars. The FFQ was also designed to gather information on participants' smoking habits. The FFQ was administered after subjects completed the multiple pass 5-step automated 24-hours dietary recall. All FFQs were analyzed for the frequency and consumption of total carbohydrate and high glycemic index (GI) foods only. The minimum frequency period for estimating total carbohydrate and high glycemic index foods was "Less than once a month", while the maximum frequency period was "Greater than five times a week". The FFQ measured total net carbohydrate and glycemic index per serving.

\subsection{Quantification of Milk Insulin}

Insulin concentration was determined by an enzymelinked immunosorbent assay (ELISA) kit (Mercodia Ul- 
tra-sensitive Insulin ELISA, 10-1132-01, Windham, NH, USA). According to the manufacturer, sensitivity of this assay is $0.07 \mathrm{mU} / \mathrm{L}$. Intra-assay $\mathrm{CV}$ was $4.3 \%$ and inter-assay $\mathrm{CV}$ was $6.8 \%$. Assays were performed in accordance with the manufacturer's instructions, in 96-well plates. Frozen milk samples were thawed in a refrigerator for two hours and vortexed briefly before use. Insulin in milk samples was assayed in duplicate by the addition of $25 \mu \mathrm{L}$ of sample. Following incubation, the microtiter plates were washed six times. This was followed by the addition $100 \mu \mathrm{L}$ of substrate to each well. After 15 minutes, the color was developed through the addition of the stop solution to each well. Then, concentration of insulin standards and insulin in the milk samples were read at $450 \mathrm{~nm}$ using an automated plate reader (Sigma Diagnostics EIA MultiWell Reader II; Owner's Manual Document 2101, St Louis, MO).

\subsection{Quantification of Milk Glucose}

Glucose concentrations were quantified enzymatically using the VITROS 250 Chemistry System Analyzer (Ortho-Clinical Diagnostics, Rochester, NY, USA) using glucose oxidase spectrophotometric dry reagent chemistry. Assay sensitivity is stated as $10 \mathrm{mg} / \mathrm{dL}$ of glucose. Inter-assay precision was $1.7 \%$ and intra-assay precision was $1.4 \%$. A $10 \mu \mathrm{L}$ breast-milk sample was deposited on a dry reagent slide. Milk glucose was catalyzed by glucose oxidase to form d-gluconic acid and hydrogen peroxide. This reaction was followed by an oxidative coupling catalyzed by peroxidase in the presence of dye precursors to produce a red dye. The intensity of this reaction was measured by reflected light. Glucose concentration was directly proportional to the amount of reflected light. The VITROS GLU Slide method uses a calorimetric type test. Its approximate incubation time is 5 minutes at $37^{\circ} \mathrm{C}\left(98.6^{\circ} \mathrm{F}\right)$. The test is determined at a wavelength of $540 \mathrm{~nm}$.

\subsection{Statistical Methods}

Statistical analysis was performed using the Statistical Package for Social Sciences (SPSS) software (SPSS for Windows version 14.0, 2005, SPSS Inc, Chicago, IL). Descriptive statistics were used to describe and summarize the nature of the sample obtained in this study.

In preliminary analyses, distributions of study variables were examined for missing values, outliers, and violations to the normality assumption. There were no missing data, and we found no extreme skewness (i.e. less than -2 or greater than 2). One univariate outlier was found for BMI of a mother in Group 2, but was retained in the study as skewness did not relate to a serious departure from the normal distribution. Mean differences in levels of insulin and glucose in the breast-milk of the two groups of mothers were assessed using independentsamples $t$ tests. Pearson's correlation coefficient $(r)$ was used to assess inter-relationships among study variables. Statistical significance was set $a$ priori at the level of $p \leq$ 0.05 .

\section{Results}

We predicted that levels of insulin and glucose in the milk of overweight/obese mothers would be greater than those of normal weight mothers. We also expected to find pre-pregnancy BMI inter-related with milk-glucose and milk-borne insulin levels.

\subsection{Demographic Characteristics of Participants}

A total of 32 participants completed the study. Twentyone participants were in Group 1 (non-obese), 11 were in Group 2 (overweight/obese). The mean age was 27.42 years $(\mathrm{SD}=6.16$; range $=18-36)$ for mothers in Group 1 , and 27.36 years $(\mathrm{SD}=4.69$; range $=20-39)$ for mothers in Group 2. The two groups did not differ in age, $t(30)=0.030, p=0.976$.

The mean BMI for Group 1 was $21.80 \mathrm{~kg} / \mathrm{m}^{2}(\mathrm{SD}=$ 2.34 ; range $\left.=(18.5-24.7) \mathrm{kg} / \mathrm{m}^{2}\right)$ while, that for Group 2 was $32.23 \mathrm{~kg} / \mathrm{m}^{2}(\mathrm{SD}=3.82 ;$ range $=(26.40-40.20)$ $\mathrm{kg} / \mathrm{m}^{2}$ ). BMI of women in Group 2 was greater than that of women in Group 1, $t(30)=9.582, p=0.001$.

\subsection{Concentrations of Breast-Milk Glucose and Mothers' BMI}

The mean concentration of glucose in the breast-milk of overweight/obese mothers was $(51.9 \pm 20.5) \mathrm{mg} / \mathrm{dL}$, whereas that of normal weight mothers was $(32.6 \pm 21.8)$ $\mathrm{mg} / \mathrm{dL}$ (Figure 1). An independent-samples $t$ test revealed that the glucose level of overweight/obese women's breast-milk was greater than that of normal weight women, $t(30)=2.43, p=0.02$. Pearson's correlation analysis yielded a significant $(r=0.483, p<0.005)$ association between breast-milk glucose level and pre-pregnancy BMI.

\subsection{Concentrations of Breast-Milk Insulin and Mothers' BMI}

The mean concentration of insulin in the breast-milk of overweight/obese mothers was $(30.1 \pm 56.3) \mu \mathrm{IU} / \mathrm{mL}$, whereas that of normal weight mothers was $(4.5 \pm 7.6)$ $\mu \mathrm{IU} / \mathrm{mL}$ (Figure 2). An independent-samples $t$ test indicated that the concentration of milk insulin differed between the two groups, $t(30)=2.075, p=0.05$. A Pearson correlation analysis revealed a positive association between pre-pregnancy BMI and level of breast-milk insu$\operatorname{lin}(r=0.565, p<0.001)$.

Taken together, findings on glucose and insulin revealed that in comparison with women with normal pre-preg- 


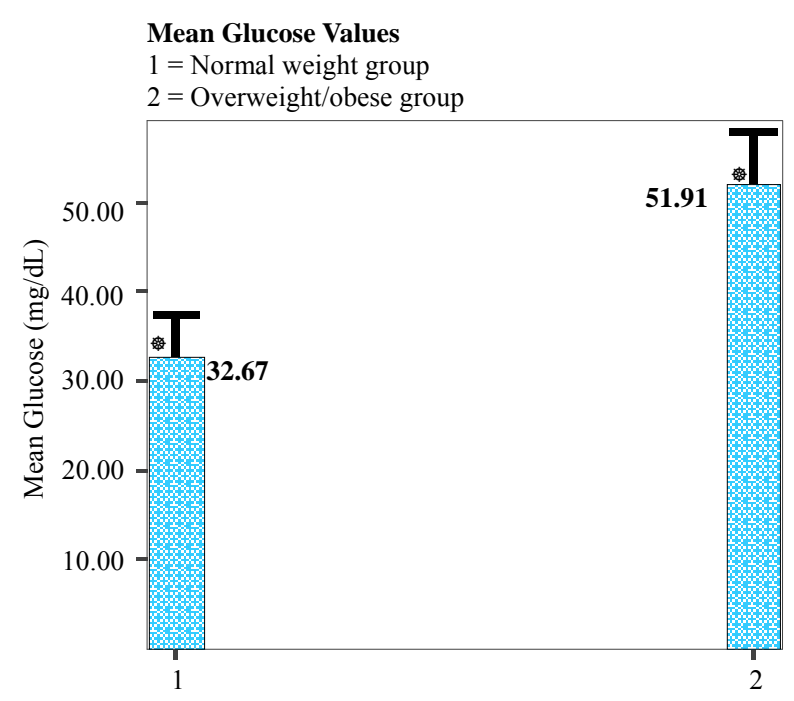

Figure 1. Mean levels of glucose in breast-milk of normal weight and overweight/obese mothers, Error bars show +1 SEM, $1 \mathrm{mg} / \mathrm{dL}$ glucose $=0.0555 \mathrm{mmol} / \mathrm{L}$.

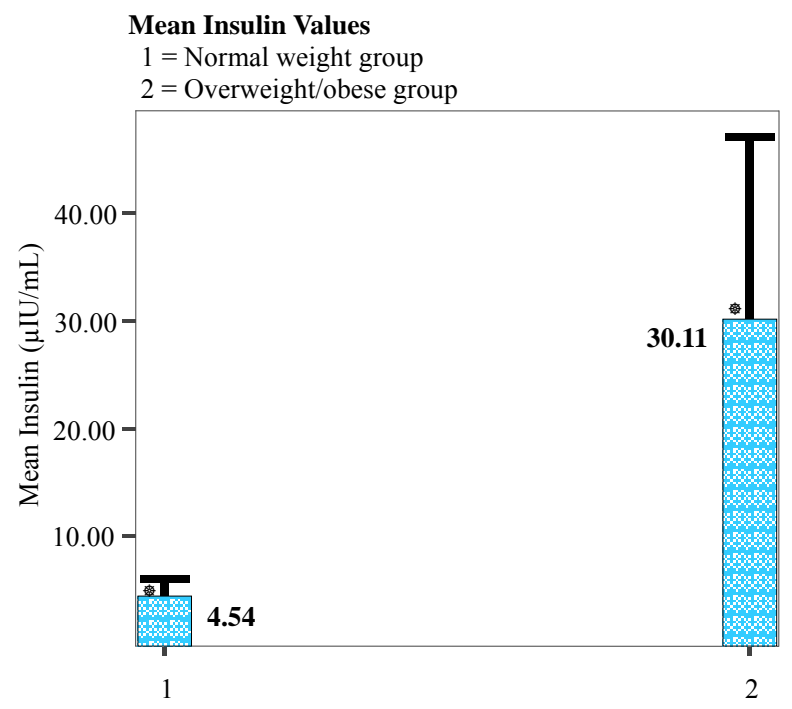

Figure 2. Mean levels of insulin in breast-milk of normal weight and overweight/obese mothers, Error bars show +1 SEM, $1 \mu \mathrm{IU} / \mathrm{mL}$ insulin $=6.945 \mathrm{pmol} / \mathrm{L}$.

nancy BMI, those who were overweight/obese had elevated levels of glucose and insulin in their breast-milk, and these levels were inter-correlated with pre-pregnancy BMI.

\subsection{Effect of Maternal Dietary Intake on Levels of Glucose and Insulin in Breast-Milk}

Pearson's correlation analyses $(r)$ were used to determine whether levels of glucose and insulin in breast-milk were associated with total kilocalories, carbohydrates, sugars, and fats consumed by mothers during the 24-hour period prior to milk sample collection. These failed to uncover any significant associations (data not shown). Independent-samples $t$ tests revealed that dietary patterns, in terms of total kilocalories, carbohydrates, sugars, and fats, did not differ between groups, ruling out the effects of dietary intake as confounding influences on breast-milk glucose and insulin.

Data obtained from the 24-hour dietary recall were also used to derive an average daily estimated glycemic index (EGI). Pearson's correlation ( $r$ ) analysis was used to determine the relationship between the average daily EGI and total carbohydrate and sugar consumed during the 24-hour period prior to sample collection. This yielded a positive association $(r=0.364, p<0.05)$ between average daily EGI and total sugars. However, relationships between average daily EGI and levels of glucose and insulin in breast-milk were non-significant.

\section{Discussion}

Although breast-milk is known to contain important compounds, including glucose and insulin [9-11], it still remains unclear whether milk from an obese or a diabetic mother contains these compounds at levels greater than those found in mothers with a normal BMI. Infants born to obese mothers are at an increased risk of developing diabetes as well as impaired glucose tolerance during childhood, and this trend may continue through adulthood [3]. The present study found that at six weeks postpartum, levels of glucose and insulin in the breastmilk of overweight/obese mothers were higher than those of normal weight mothers. Also, maternal pre-pregnancy BMI and levels of glucose and insulin in breast-milk were inter-related. Researchers have reported that both glucose and insulin levels in human breast-milk appear to be higher in diabetic women as compared to non-diabetic women [12]. Additionally, concentrations of glucose in the milk of diabetic women have been found higher than those in the milk of non-diabetic women [13]. Glucose is the major substrate for fat synthesis. In the presence of metabolic abnormalities due to obesity and diabetes, there are changes in metabolism that lead to an increase in glucose. These, in-turn, lead to changes in insulin in plasma as well as breast-milk [13]. Women with type 2 and gestational diabetes have generally been found to be overweight/obese, so it could be that the higher levels of glucose and insulin in their breast-milk, as reported by the present and previous studies, may be more closely related to their BMI [14].

Of several bioactive compounds found in human milk, some, including glucose, appear to be rapidly transported into breast-milk directly from maternal circulation without undergoing any changes with respect to their struc- 
ture or activity levels and patterns [15]. Experimental studies with rats have shown that large peptides, such as insulin, may be passing across the intestinal epithelium of the suckling pups from maternal milk. However, it remains unclear whether insulin from mother's milk has any effect on the peripheral organs, such as the pancreas, of the suckling rats. Well before the findings of Kinouchi et al. [16], studies revealed that insulin is found at higher concentrations in milk as compared to blood in most mammals, including humans $[15,17]$. In fact, within a few days of lactation, insulin concentrations in human milk appear to taper off and correlate proportionally to concentrations found in blood $[15,17]$. It has been speculated that even a slight increase in glucose in breast-milk may have long-term repercussions, such as increased weight gain and altered metabolism in infants [3]. In the present study, differences in glucose and insulin levels in the breast-milk of overweight/obese versus normal weight mothers partly confirms the hypothesis that high maternal pre- pregnancy BMI relates to some biological activities in a lactating mother with potential to affect the nursing infant's weight, and predispose them to obesity and related disorders in the future.

In the present study, dietary assessments were completed to help control for extraneous variables, such as, high carbohydrate intake prior to milk sample collection, which inadvertently may have had some effect on glucose and insulin levels in breast-milk. Even a moderate increase $(55 \%)$ in maternal nutrient intake above the recommended dietary allowance for pregnant women causes concentrations of maternal plasma glucose and insulin to increase simultaneously [18]. In the current study, maternal dietary intake, as assessed by a 24 -hour diet recall and FFQ, was not found correlated with glucose or insulin in breast-milk. This leads to suggesting that the levels of glucose and insulin that we detected in breast-milk were independent of dietary carbohydrate and simple sugar consumption. A limitation of this observation is that maternal dietary intake was obtained via self-reporting, which may have limited the interpretation of the data and the outcomes. Additionally, nonsignificant results could have been due to the limited sample size. A larger sample may have produced greater power and possibly different outcomes. Ratzman et al. [19] studied glucose concentration in breast-milk of 11 nursing diabetic mothers and 11 aged-matched control subjects. This study revealed that plasma glucose concentrations and glycosylated hemoglobin A1 had no effect on glucose concentrations in breast-milk. Those results are relevant to the interpretation of the present study's results. Although, unlike Ratzman et al. [19], we were unable to include blood glucose analysis to substantiate our find- ings on breast-milk, it is reasonable to assume that the conclusions offered by Ratzman et al. [19] support our conjecture that high BMI may be a contributing factor that is responsible for the higher levels of glucose and insulin found in breast-milk. Further support for this assertion may be drawn from our finding that it seems unlikely that high glucose and insulin found in breast-milk were due to high carbohydrate consumption by mothers immediately prior to milk sample collection since there were no differences between groups on these variables.

The present study was limited by a number of factors. While adding rigor and controls, the inclusion/exclusion criteria (including exclusive breast-feeding and timing of breast milk collection), resulted in a sampling frame that was very specific and limited. Consequently, generalizations of the findings of this study are limited to the criteria used for this study. Breast-feeding mothers who enrolled in this study agreed to provide the researcher with a detailed record of their entire food intake for the 24-hour period prior to milk sample collection and additional dietary information. However, the primary limitation of using these instruments to gather dietary information is their reliance on self-reporting. Milk samples were analyzed for levels of glucose and insulin at only one point in time, six-weeks postpartum. It would be interesting to explore levels of such compounds in milk over several time-periods during the course of lactation. Finally, the study was limited by the absence of analyses of blood for determining levels of glucose and insulin. Our sole reliance on breast-milk was driven by hospital policies which precluded blood work being conducted on women with high BMI as these women are classified as "high-risk". Future work which includes analyses of blood and estimation of physical activity is needed. Patterns of infant feeding have been linked with adult obesity [20]. Hence, future studies investigating long-term relationship between composition of breast-milk and subsequent metabolic effects on the nursing infant are required.

\section{Conclusions}

Maternal pre-pregnancy BMI was found correlated with breast-milk glucose and insulin concentrations at six weeks postpartum. Further investigation is required toward fuller insight into the effects of maternal body composition and the impact of increased glucose and insulin in breast-milk on the nursing infant's weight and metabolism. Studies are also needed to investigate whether these differences in breast-milk components are sustained over longer durations of breast-feeding. Additional studies are also required to investigate the benefits and risks of breast-feeding among obese and diabetic 
mothers. While there are some inconsistencies in the research literature, this study adds groundwork to explore the relationships between maternal body composition and its potential impact on the lactating mother and her nursing infant.

\section{REFERENCES}

[1] R. Locke, "Preventing Obesity: The Breast Milk-Leptin Connection," Acta Paediatr, Vol. 91, No. 9, 2002, pp. 891-894. doi:10.1111/j.1651-2227.2002.tb02871.x

[2] R. Novotny, P. Coleman, L. Tenorio, N. Davison, T. Camacho, V. Ramirez, V. Vijayadeva, P. Untalan and M. D. Tudela, "Breastfeeding Is Associated with Lower Body Mass Index among Children of the Commonwealth of the Northern Mariana Islands," Journal of the American Dietetic Association, Vol. 107, No. 10, 2007, pp. 1743-1746. doi:10.1016/j.jada.2007.07.018

[3] A. Plagemann, T. Harder, K. Franke and R. Kohlhoff, "Long-Term Impact of Neonatal Breast-Feeding on Body Weight and Glucose Tolerance in Children of Diabetic Mothers," Diabetes Care, Vol. 25, No. 1, 2002, pp. 216222. doi:10.2337/diacare.25.1.16

[4] P. M. Catalano, J. P. Kirwan, S. Haugel-de Mouzon and J. King, "Gestational Diabetes and Insulin Resistance: Role in Short- and Long-Term Implications for Mother and Fetus," The Journal of Nutrition, Vol. 133, No. 5 (Supplement), 2003, pp. 1674S-1683S.

[5] J. M. Conway, L. A. Ingwersen, B. T. Vinyard and A. J. Moshfegh, "Effectiveness of the US Department of Agriculture 5-Step Multiple-Pass Method in Assessing Food Intake in Obese and Nonobese Women," American Journal of Clinical Nutrition, Vol. 77, No. 5, 2003, pp. 1171-1178.

[6] Johns Hopkins Bayview Medical Center, "Food Frequency Questionnaire," 2007. http://www. hopkinsbayview.org/

[7] R. D. Lee and D. C. Nieman, "Nutritional Assessment," 4th Edition, McGraw Hill, NewYork, 2007.

[8] "Nutrition Data," 2007. http://www.nutritiondata.com

[9] J. Bitman, M. Hamosh, P. Hamosh, V. Lutes, Mc. Neville, J. Seacat and D. L. Wood, "Milk Composition and Volume during the Onset of Lactation in a Diabetic Mother," American Journal of Clinical Nutrition, Vol. 50, No. 6, 1989, pp. 1364-1369.

[10] Y. O. Ilcol, Z. B. Hizli and T. Ozkan, "Leptin Concentration in Breast Milk and Its Relationship to Duration of
Lactation and Hormonal Status," International Breastfeeding Journal, Vol. 1, No. 21, 2006.

[11] C. J. Stocker, J. R. Arch and M. A. Cawthorne, "Fetal Origins of Insulin Resistance and Obesity," Proceedings of the Nutrition Society, Vol. 64, No. 2, 2005, pp. 143 151. doi:10.1079/PNS2005417

[12] L. Jovanovic-Peterson, K. Fuhrmann, K. Hedden, L. Walker and C. M. Peterson, "Maternal Milk and Plasma Glucose and Insulin Levels: Studies in Normal and Diabetic Subjects," Journal of the American College of Nutrition, Vol. 8, No. 2, 1989, pp. 125-131.

[13] N. F. Butte, C. Garza, R. Burr, A. S. Goldman, K. Kennedy and J. L. Kitzmiller, "Milk Composition of Insulin-Dependent Diabetic Women," Journal of Pediatric Gastroenterology \& Nutrition, Vol. 6, No. 6, 1987, pp. 936-941. doi:10.1097/00005176-198711000-00020

[14] N. Marcia, K. P. Sucher and S. Long, "Nutrition Therapy and Pathophysiology," Brooks Cole, Belmont, 2007.

[15] C. E. Grosvenor, M. F. Picciano and C. R. Baumrucker, "Hormones and Growth Factors in Milk," Endocrine Reviews, Vol. 14, No. 6, 1993, pp. 710-728.

[16] T. Kinouchi, K. Koizumi, T. Kuwata and T. Yajima, "Crucial Role of Milk-Borne Insulin in the Development of Pancreatic Amylase at the Onset of Weaning in Rats," American Journal of Physiology, Vol. 275, No. 6, 1998, pp. R1958-1967.

[17] G. J. Ebrahim, "Breastmilk Endocrinology," Journal of Tropical Pediatrics, Vol. 42, No. 1, 1996, pp. 2-4. doi:10.1093/tropej/42.1.2

[18] I. C. McMillen, B. S. Muhlhausler, J. A. Duffield and B. S. Yuen, "Prenatal Programming of Postnatal Obesity: Fetal Nutrition and the Regulation of Leptin Synthesis and Secretion before Birth," Proceedings of the Nutrition Society, Vol. 63, No. 3, 2004, pp. 405-412. doi:10.1079/PNS2004370

[19] K. P. Ratzmann, E. Steindel, R. Hildebrandt and R. Kohlhoff, "Is There a Relationship between Metabolic Control and Glucose Concentration in Breast Milk of Type 1 (Insulin-Dependent) Diabetic Mothers?" Experimental and Clinical Endocrinology \& Diabetes, Vol. 92, No. 1, 1988, pp. 32-36. doi:10.1055/s-0029-1210778

[20] D. J. Pettitt, M. R. Forman, R. L. Hanson, W. C. Knowler and P. H. Bennett, "Breastfeeding and Incidence of NonInsulin-Dependent Diabetes Mellitus in Pima Indians," Lancet, Vol. 350, No. 9072, 1997, pp. 166-168. doi:10.1016/S0140-6736(96)12103-6 